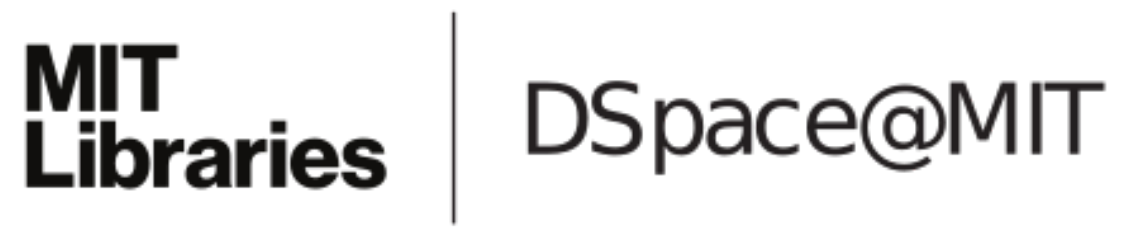

\author{
MIT Open Access Articles
}

Reverse Coherent Information

The MIT Faculty has made this article openly available. Please share how this access benefits you. Your story matters.

Citation: García-Patrón, Raúl et al. “Reverse Coherent Information.” Physical Review Letters 102.21 (2009): 210501. (C 2009 The American Physical Society.

As Published: http://dx.doi.org/10.1103/PhysRevLett.102.210501

Publisher: American Physical Society

Persistent URL: http://hdl.handle.net/1721.1/51755

Version: Final published version: final published article, as it appeared in a journal, conference proceedings, or other formally published context

Terms of Use: Article is made available in accordance with the publisher's policy and may be subject to US copyright law. Please refer to the publisher's site for terms of use. 


\title{
Reverse Coherent Information
}

\author{
Raúl García-Patrón, Stefano Pirandola, Seth Lloyd, and Jeffrey H. Shapiro \\ Research Laboratory of Electronics, Massachusetts Institute of Technology, Cambridge, Massachusetts 02139, USA
}

(Received 3 August 2008; published 29 May 2009)

\begin{abstract}
In this Letter we define a family of entanglement distribution protocols assisted by feedback classical communication that gives an operational interpretation to reverse coherent information, i.e., the symmetric counterpart of the well-known coherent information. This leads to the definition of a new entanglement distribution capacity that exceeds the unassisted capacity for some interesting channels.
\end{abstract}

DOI: 10.1103/PhysRevLett.102.210501

PACS numbers: 03.67. $-\mathrm{a}$

Shannon's great result was proving that sending information through a noisy channel $\mathcal{N}$ can be achieved with a vanishing error, in the limit of many uses of the channel [1]. Shannon's key idea was to add redundancy to the message in order to compensate for the channel's noise. He showed that the channel's communication capacity $\mathcal{C}(\mathcal{N})$ between two partners, called Alice and Bob, is given by the maximal mutual information between Alice's input $a$ and Bob's output $b=\mathcal{N}(a)$, i.e.,

$$
\mathcal{C}(\mathcal{N})=\max _{a} H(a: b) \quad \text { (bits/channel use) }
$$

Quantum information theory [2] is a generalization of Shannon's information theory that has attracted huge interest in the last decade, as it allows for new potential applications, such as quantum communication and entanglement distribution. Quantum communication allows faithful transfer of quantum states through a quantum noisy channel $\Lambda$. The quantum communication capacity $\mathcal{Q}(\Lambda)$ gives the number of qubits per channel use that can be reliably transmitted, preserving quantum coherence. It was shown in [3] that the coherent information $I\left(\Lambda, \rho_{A}\right)$, a function of Alice's input $\rho_{A}$ on channel $\Lambda$, plays a crucial role in the definition of the quantum communication capacity. The coherent information is

$$
I\left(\Lambda, \rho_{A}\right)=I\left(I_{R} \otimes \Lambda\left(|\psi\rangle\left\langle\left.\psi\right|_{R A}\right)\right)=I\left(\rho_{R B}\right),\right.
$$

where $|\psi\rangle_{R A}$ is the purification of $\rho_{A}$ and $I\left(\rho_{R B}\right)=S(B)-$ $S(R B)$, where $S(X)$ is the von Neumann entropy of $\rho_{X}$. By analogy with Shannon's theory, one would expect $\mathcal{Q}(\Lambda)$ to be calculated by maximizing over a single use of the channel,

$$
\mathcal{Q}^{(1)}(\Lambda)=\max _{\rho_{A}} I\left(\Lambda, \rho_{A}\right) .
$$

Unfortunately, the quantum case is more complicated, as $\mathcal{Q}^{(1)}(\Lambda)$ is known to be nonadditive [4]. The correct capacity definition [5] is,

$$
\mathcal{Q}(\Lambda)=\lim _{n \rightarrow \infty} \frac{1}{n} \max _{\rho_{\bar{A}}} I\left(\Lambda^{\otimes n}, \rho_{\bar{A}}\right) .
$$

Only for the restricted class of degradable channels [6], is
$\mathcal{Q}(\Lambda)$ known to be additive, i.e., $Q(\Lambda)=Q^{(1)}(\Lambda)$. The channel $\Lambda$ is called degradable if there exists a map $\mathcal{M}$ that transforms Bob's output $\rho_{B}$ into the environment state $\rho_{E}$, i.e., $\mathcal{M}\left(\rho_{B}\right)=\rho_{E}$, where $\rho_{E}=\operatorname{Tr}_{R B}\left[|\phi\rangle\left\langle\left.\phi\right|_{R B E}\right]\right.$ and $|\phi\rangle_{R B E}$ is the purification of $\rho_{R B}$. Similarly if there is a map $G$ such that $G\left(\rho_{E}\right)=\rho_{B}$ the channel is called antidegradable and $\mathcal{Q}(\Lambda)=0$.

Having free access to a classical communication channel Alice and Bob can improve the quantum communication protocol, as opposed to Shannon's theory where using feedback gives no improvement [7]. One can define three new quantum communication capacities depending on the use of the classical channel: forward classical communication $\left(Q_{\rightarrow}\right)$; feedback classical communication $\left(Q_{\leftarrow}\right)$; two-way classical communication $\left(Q_{\leftrightarrow}\right)$. In Fig. 1 we review the relations between these four capacities.

Entanglement is another important resource for quantum information processing. Therefore, the study of the entan-

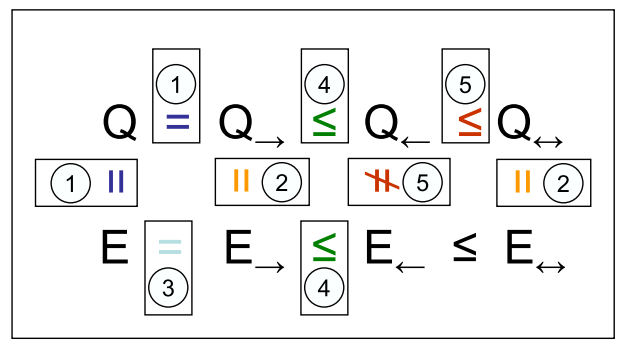

FIG. 1 (color online). Relations between the quantum communication and entanglement distribution capacities. We first start by two general remarks: (I) Being able to send a noiseless qubit is a stronger resource than distributing units of entanglement (e-bits): $\mathcal{E}_{x} \geq \mathcal{Q}_{x}$ for all $x$. (II) Increasing the complexity of the assistance cannot decrease the capacity: $\mathcal{X} \leq \chi_{\leftarrow} \leq \mathcal{X}_{\leftarrow}$. The following remarks concern their corresponding number on the figure. (1) The equality $\mathcal{E}=\mathcal{Q}=\mathcal{Q} \rightarrow$ was shown in [12]. (2) Any entanglement distribution protocol with free forward classical communication can be transformed into a quantum communication protocol by appending teleportation to it. (3) Results from combining 1 and 2. (4) Combining 1, 3 and II. (5) It is easy to prove that $\mathcal{E}_{\leftarrow}=\mathcal{Q}_{\hookleftarrow}$ for the erasure channel $[13,14]$. In [14] it was shown that the erasure channel satisfies the strict inequality $\mathcal{Q}_{\leftarrow}<\mathcal{Q}_{\hookleftarrow}$, which gives $\mathcal{E}_{\leftarrow} \neq \mathcal{Q}_{\leftarrow}$. 
glement distribution capacity of quantum channels (distributed $e$-bits per use of the channel) is of crucial importance. As for quantum communication, we can also define four types of assisted (unassisted) capacities for entanglement distribution: $\left\{\mathcal{E}_{,} \mathcal{E}_{\rightarrow}, \mathcal{E}_{\hookleftarrow}, \mathcal{E}_{\hookrightarrow}\right\}$. As shown in Fig. 1, all the entanglement distribution capacities are equivalent to their quantum communication counterparts, except for $\mathcal{E}_{\leftarrow}(\Lambda)$

Entanglement distribution assisted by feedback classical communication. - The entanglement distribution protocol assisted by classical feedback communication, as described in [8], goes as follows. Alice starts preparing a bipartite entangled state $\Psi_{R \mid A_{1}, A_{2}, \ldots, A_{n}}$, where $R$ is a group of qubits entangled with the qubits $A_{i}$ sent, one by one, through the channel $\Lambda$. The first round of the protocol, see Fig. 2, consists of three steps: (i) Alice sends qubit $A_{1}$ through the quantum channel $\Lambda$; (ii) Bob applies an incomplete quantum measurement $\mathcal{B}_{1}$ over his received qubit $B_{1}$ and communicates the classical outcome $b_{1}$ to Alice; (iii) Alice, condition on the classical message $b_{1}$, applies a global quantum operation $\mathcal{A}_{1}^{b_{1}}$ over the joint system of $R$ and the remaining $n-1$ qubits $A_{2} A_{3} \ldots A_{n}$. The next $n-1$ rounds are a slight modification of the first one. First, Bob's measurement $\mathcal{B}_{i}^{b_{1} \ldots b_{i-1}}$ acts on all his received qubits $B_{1} B_{2} \ldots B_{i}$, conditioned on his previous measurement outcomes $b_{1} \ldots b_{i-1}$. Second, Alice's operation $\mathcal{A}_{i}^{b_{1} \ldots b_{i}}$, acts on all her remaining qubits $R A_{i+1} \ldots A_{n}$, conditioned on all previous classical communication messages. By properly choosing Alice's operations and Bob's incomplete measurements both partners extract $\approx n \mathcal{E}_{\leftarrow}(\Lambda)$ units of entanglement ( $e$-bits) at the end of the protocol. Unfortunately, the calculation of $\mathcal{E}_{\leftarrow}(\Lambda)$ is extremely challenging in full generality.

Reverse entanglement distribution.-A big practical disadvantage of the previous protocol is that Alice has to wait

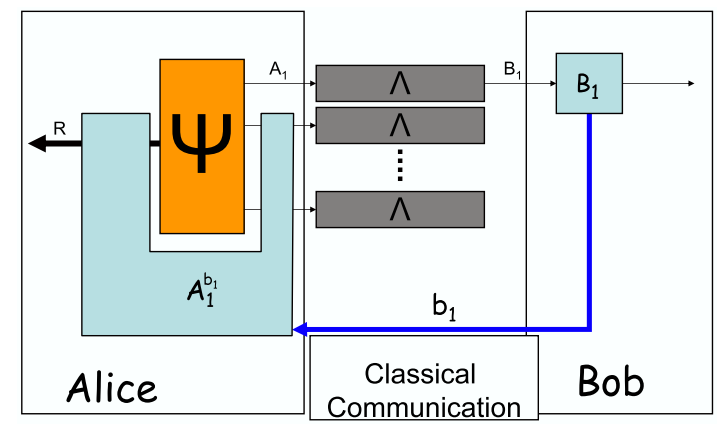

FIG. 2 (color online). The first round of the entanglement distribution protocol assisted by classical feedback consists of three steps: (i) Alice sends qubit $A_{1}$ through the quantum channel $\Lambda$; (ii) Bob applies an incomplete quantum measurement $\mathcal{B}_{1}$ over his received qubit $B_{1}$ and communicates the outcome $b_{1}$ to Alice; (iii) Alice applies a global quantum operation $\mathcal{A}_{1}^{b_{1}}$ over the joint system of $R$ and the remaining $n-1$ qubits $A_{i}$. The next rounds are straightforward extensions of the first one. until Bob sends the message $b_{i}$ before applying $\mathcal{A}_{i}^{b_{1} \ldots b_{i}}$ and subsequently sending qubit $A_{i+1}$, which greatly decreases the transmission rate. A way of avoiding this problem is to simplify the protocol to a single round of classical feedback after Alice has sent all her qubits $A_{1} A_{2} \ldots A_{n}$ through the quantum channel $\Lambda$; see Fig. 3. We call this familly of simplified protocols reverse entanglement distribution protocols, by analogy with the quantum key distribution scenario [9]. Before the single postprocessing round Alice and Bob's shared state is

$$
\rho_{R \mid B_{1}, B_{2}, \ldots, B_{n}}=I_{R} \otimes \Lambda^{\otimes n}\left(\Psi_{R \mid A_{1}, A_{2}, \ldots, A_{n}}\right) .
$$

By properly choosing Alice's and Bob's operations both partners extract $\approx n \mathcal{E}_{\triangleleft}(\Lambda) e$-bits, where $\mathcal{E}_{\triangleleft}(\Lambda)$ is the reverse entanglement distribution capacity, satisfying the inequality $\mathcal{E}_{\triangleleft}(\Lambda) \leq \mathcal{E}_{\leftarrow}(\Lambda)$.

Remark that, in the particular case where Alice's inputs are independent and identically distributed, i.e., $\rho_{R \mid A_{1}, A_{2}, \ldots, A_{n}}=\rho_{R \mid A}^{\otimes n}$, the postprocessing of the reverse entanglement distribution protocol is the dynamical equivalent of an entanglement distillation protocol over the static resource $\rho_{R \mid B}^{\otimes n}[10]$.

Reverse coherent information capacity.-In what follows we consider a subset of the reverse entanglement distribution protocols with a strikingly simple capacity that lower bounds $\mathcal{E}_{\triangleleft}(\Lambda)$. By exchanging the roles of Alice and Bob in the family of static distillation protocol assisted by one-way classical communication defined in [10], we obtain a new family of static distillation protocols with rate

$$
I_{R}\left(\rho_{R B}\right)=S(R)-S(R B) .
$$

By analogy with the quantum key distribution scenario [9], we call the quantity $I_{R}\left(\rho_{R B}\right)$ the reverse coherent information. It is then straightforward to consider a family of

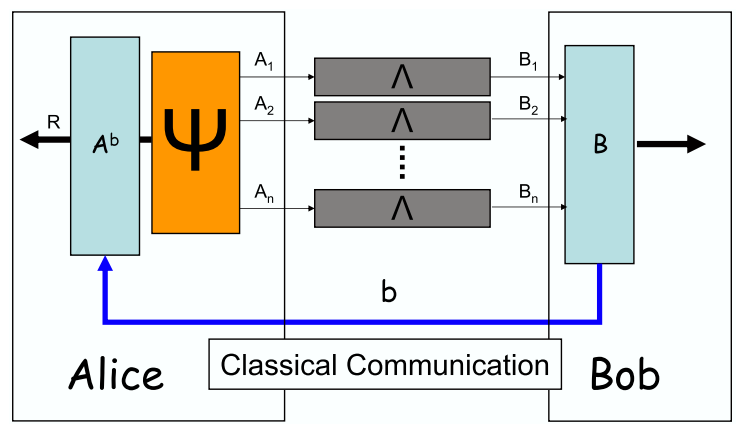

FIG. 3 (color online). A simplification of the general entanglement distribution protocol assisted by classical feedback (Fig. 2) limits the protocol to a last single round of processing. After Alice has sent all her qubits $\left(A_{1} A_{2} \ldots A_{n}\right)$ through the quantum channel $\Lambda$, Bob applies a collective incomplete measurement $\mathcal{B}$ among all the qubits $B_{1} B_{2} \ldots B_{n}$ and communicates the classical outcome $b$ to Alice. Finally, conditioned on the message $b$, Alice applies the quantum operation $\mathcal{A}^{b}$ on system $R$. 
entanglement distribution protocols assisted by classical feedback with rate $I_{R}\left(\Lambda, \rho_{A}\right)=I_{R}\left(I_{R} \otimes \Lambda\left(|\psi\rangle\left\langle\left.\psi\right|_{R A}\right)\right)=\right.$ $I_{R}\left(\rho_{R B}\right)$. Optimizing this rate over $\rho_{A}$ we define the singleletter reverse coherent information capacity $\mathcal{E}_{R}^{(1)}(\Lambda)$.

Similarly to Eq. (4) we can define a regularized entanglement capacity $\mathcal{E}_{R}(\Lambda)$ that lowerbounds $\mathcal{E}_{\triangleleft}(\Lambda)$. Interestingly, this quantity can be shown to be additive for all channels, i.e., $\mathcal{E}_{R}=\mathcal{E}_{R}^{(1)}$. To do so we only need to prove the relation

$$
I_{R}\left(\Lambda \otimes \Lambda, \rho_{A_{1} A_{2}}\right) \leq I_{R}\left(\Lambda, \rho_{A_{1}}\right)+I_{R}\left(\Lambda, \rho_{A_{2}}\right) .
$$

Using the alternative definition of the reverse coherent information $I_{R}\left(\rho_{R B}\right)=S(B E)-S(E)$, where $|\phi\rangle_{R B E}$ is the purification of $\rho_{R B}$ and $\rho_{B E}$, Eq. (7) can be restated as a relation between two von Neumann mutual information quantities: $S\left(B_{1} E_{1}: B_{2} E_{2}\right) \geq S\left(E_{1}: E_{2}\right)$. This relation holds because discarding quantum systems can only decrease the mutual information, which results from the strong-subadditivity of the entropy.

The previous proof is strikingly similar to the additivity of the unassisted capacity of degradable channels, except that it holds for all channels. Since $I_{R}\left(\Lambda, \rho_{A}\right)$ is additive, it would be extremely interesting if it could be used to give a definition of $\mathcal{E}_{\leftarrow}(\Lambda)$ or $\mathcal{E}_{\triangleleft}(\Lambda)$ similar to Eq. (4). Unfortunately, this cannot be done as $I_{R}\left(\Lambda, \rho_{A}\right)$ does not satisfy the data processing inequality.

Despite reverse coherent information capacity restricts the protocols to a very specific subset, its study remains very interesting, as for some channels it achieves a remarkable improvements over the unassisted capacity $\mathcal{E}(\Lambda)$. To get some intuition on when we may obtain an improvement, we look at the difference between the coherent information and its reverse counterpart $\left(I_{R}\left(\rho_{R B}\right)-\right.$ $\left.I\left(\rho_{R B}\right)=S(R)-S(B)\right)$. We see that for channels satisfying $S(R)>S(B)$ over all inputs, such as the bosonic lossy channel, reverse reconciliation performs better than $\mathcal{E}^{(1)}$. On the other hand, for those channels satisfying $S(B) \geq$ $S(R)$ for all inputs, such as optical amplifiers or the erasure channel, we obtain $\mathcal{E} \geq \mathcal{E}^{(1)} \geq \mathcal{E}_{R}$. In the case of the erasure channels is it easy to see that $\mathcal{E}_{\leftrightarrow}=\mathcal{E}_{\triangleleft}>\mathcal{E}>$ $\mathcal{E}_{R}$, which gives an example of strict separation between $\mathcal{E}_{\triangleleft}$ and $\mathcal{E}_{R}$.

Amplitude damping channel.-The amplitude damping channel describes the process of energy dissipation through spontaneous emission in a two-level system. The effect of the channel on the input state $\rho$ is $\mathcal{D}_{\eta}(\rho)=$ $E_{0} \rho E_{0}^{\dagger}+E_{1} \rho E_{1}^{\dagger}$, where

$$
E_{0}=\left[\begin{array}{cc}
1 & 0 \\
0 & \sqrt{\eta}
\end{array}\right], \quad E_{1}=\left[\begin{array}{cc}
0 & \sqrt{1-\eta} \\
0 & 0
\end{array}\right],
$$

and $1-\eta$ is the probability of spontaneous emission. Generalizing the results of [11], we can restrict the input state to the class $\rho_{A}=\operatorname{diag}(1-p, p)$ without loss of generality. For a given input population $p$, the output state is
$\rho_{B}=\operatorname{diag}(1-\eta p, \eta p)$ and the (reverse) coherent information becomes

$$
\begin{aligned}
I(\mathcal{E}, p) & =H(\eta p)-H((1-\eta) p), \\
I_{R}(\mathcal{E}, p) & =H(p)-H((1-\eta) p),
\end{aligned}
$$

where $H(x)$ is the binary entropy. Optimizing over the input population we obtain $\mathcal{E}^{(1)}\left(\mathcal{D}_{\eta}\right)$ and $\mathcal{E}_{R}\left(\mathcal{D}_{\eta}\right)$ as functions of the damping parameter $\eta$; see Fig. 4. Using the concatenation property of the amplitude damping channel $\left(\mathcal{D}_{\eta} \circ \mathcal{D}_{\eta^{\prime}}=\mathcal{D}_{\eta \eta^{\prime}}\right)$ it is easy to prove that the amplitude damping channel is degradable $\left(\mathcal{E}\left(\mathcal{D}_{\eta}\right)=\mathcal{E}^{(1)}\left(\mathcal{D}_{\eta}\right)\right)$ for $\eta \geq 1 / 2$ and antidegradable $\left(\mathcal{E}\left(\mathcal{D}_{\eta}\right)=0\right)$ for $\eta \leq 1 / 2$. We conclude that $\mathcal{E}_{R}\left(\mathcal{D}_{\eta}\right)$ outperforms $\mathcal{E}\left(\mathcal{D}_{\eta}\right)$ for all $\eta$. Even more interestingly, $\mathcal{E}_{R}\left(\mathcal{D}_{\eta}\right)$ remains positive in the range $\eta \leq 1 / 2$ where $\mathcal{E}\left(\mathcal{D}_{\eta}\right)=0$; see Fig. 4(a).

Generalized amplitude damping channel.-Spontaneous emission to an environment at thermal equilibrium leads to the generalized amplitude damping channel $\mathcal{D}_{(\eta, \alpha)}$, which can be modeled by the Stinespring's dilation circuit of Fig. 5. The relaxation operation applies the unitary transformation,

$$
U_{\mathrm{RO}}=\left[\begin{array}{cccc}
1 & 0 & 0 & 0 \\
0 & \sqrt{\eta} & \sqrt{1-\eta} & 0 \\
0 & -\sqrt{1-\eta} & \sqrt{\eta} & 0 \\
0 & 0 & 0 & 1
\end{array}\right],
$$

jointly to the input state and the environment. The thermal environment is modeled by inserting half of an entangled state $\left|\Psi_{\alpha}\right\rangle=\sqrt{1-\alpha}|00\rangle+\sqrt{\alpha}|11\rangle$ into the second input of $U_{\mathrm{RO}}$. The channel can be seen as the random mixing $\mathcal{D}_{(\eta, \alpha)}=\alpha \mathcal{D}_{(\eta, 0)}+(1-\alpha) \mathcal{D}_{(\eta, 1)}$ of two limiting cases: (1) the amplitude damping channel when $\left(\mathcal{D}_{(\eta, 0)}\right)$; and (2) a populating channel $\left(\mathcal{D}_{(\eta, 1)}\right)$. We restrict the analysis
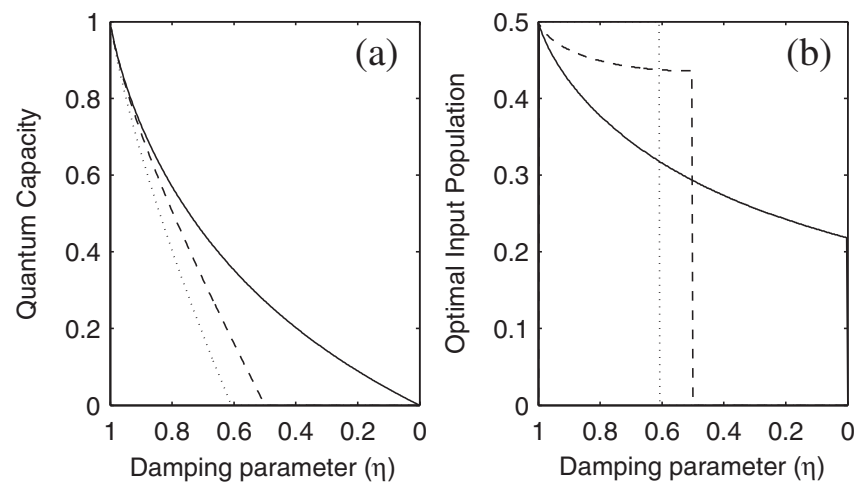

FIG. 4. (a) Comparison of $\mathcal{E}\left(\mathcal{D}_{\eta}\right)$ (dashed line) and $\mathcal{E}_{R}\left(\mathcal{D}_{\eta}\right)$ (solid line) as functions of the damping parameter $\eta$ for the amplitude damping channel, together with the capacity $\mathcal{E}^{(1)}\left(\mathcal{D}_{\eta}\right)=\mathcal{E}_{R}\left(\mathcal{D}_{\eta}\right)$ of the generalized amplitude damping channel with a maximally mixed environment $(\alpha=1 / 2)$ (dotted line). (b) Optimal input population $p$ achieving the previous capacities. 


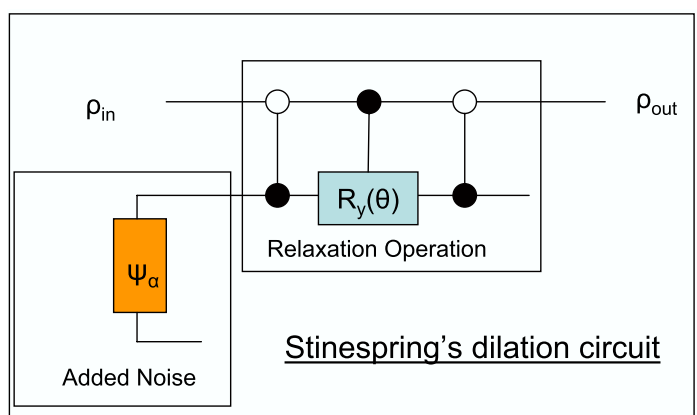

FIG. 5 (color online). Quantum circuit corresponding to the Stinespring's dilation of the generalized amplitude damping channel $\mathcal{D}_{(\eta, \alpha)}$. Alice's input state $\rho_{\text {in }}$ and half of an entangled state $\left|\Psi_{\alpha}\right\rangle$ interact through the relaxation operation $U_{\mathrm{RO}}$ composed of two CNOT gates and a controlled rotation around the $y$ axis of the Bloch sphere $\left[\cos ^{2}(\gamma / 2)=\eta\right]$.

to $0 \leq \alpha \leq 1 / 2$ as for any channel $\mathcal{D}_{(\eta, \alpha=1 / 2+x)}$ with optimal input population $p^{*}$ there is a symmetric channel $\mathcal{D}_{(\eta, \alpha=1 / 2-x)}$ with optimal population $1-p^{*}$ reaching the same capacity. As before, Alice's input can be restricted to $\rho_{A}=\operatorname{diag}(1-p, p)$ giving

$$
\begin{aligned}
& S(B)=H(\eta p+(1-\eta) \alpha), \\
& S(A B)=H_{4}\left(\lambda_{1}, \lambda_{2}, \lambda_{3}, \lambda_{4}\right),
\end{aligned}
$$

where $H_{4}$ is the Shannon entropy of a four-dimensional distribution and $\lambda_{j}$ are the four eigenvalues of $\rho_{A B}$,

$$
\begin{gathered}
\lambda_{1}=\alpha(1-\eta)(1-p), \quad \lambda_{2}=(1-\alpha)(1-\eta) p, \\
\lambda_{3,4}=\left[1-\lambda_{1}-\lambda_{2} \pm \sqrt{1-2\left(\lambda_{1}+\lambda_{2}\right)+\left(\lambda_{2}-\lambda_{1}\right)^{2}}\right] / 2 .
\end{gathered}
$$

Optimizing over the input population $p$ we obtain the
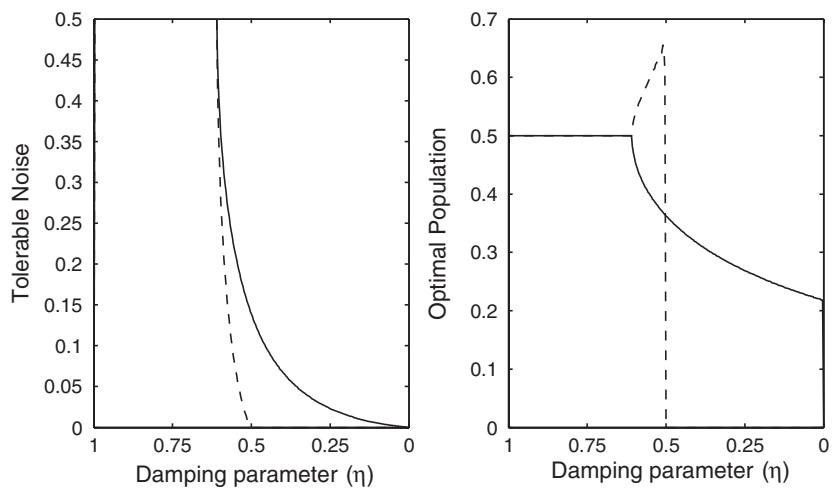

FIG. 6. (a) Tolerable thermal noise of the generalized amplitude damping channel $\mathcal{D}_{(\eta, \alpha)}$ (minimum $\alpha$ such that the capacity is zero) as a function of the damping parameter $\eta$ for: $\mathcal{E}^{(1)}\left(\mathcal{D}_{(\eta, \alpha)}\right)$ (dashed line), and $\mathcal{E}_{R}\left(\mathcal{D}_{(\eta, \alpha)}\right)$ (solid line). (b) Input population $p$ achieving the curves of (a). capacities $\mathcal{E}^{(1)}\left(\mathcal{D}_{(\eta, \alpha)}\right)$ and $\mathcal{E}_{R}\left(\mathcal{D}_{(\eta, \alpha)}\right)$. It is easy to show that $\mathcal{E}_{R}\left(\mathcal{D}_{(\eta, \alpha)}\right)>\mathcal{E}^{(1)}\left(\mathcal{D}_{(\eta, \alpha)}\right)$ for any noise $\alpha$ except for $\alpha=1 / 2$, where both are equal, as shown in Fig. 4. Unfortunately, we cannot conclude $\mathcal{E}_{R}\left(\mathcal{D}_{\eta, \alpha}\right) \geq \mathcal{E}\left(\mathcal{D}_{(\eta, \alpha)}\right)$ for $\alpha>0$, as the channels are no longer degradable. Nevertheless, it is easy to prove that generalized amplitude damping channels $\left(\mathcal{D}_{(\eta, \alpha)}\right)$ with $\eta \leq 1 / 2$ are antidegradable $\left(\rho_{B}=\mathcal{D}_{(\eta /(1-\eta), \alpha)}\left(\rho_{E}\right)\right)$, which shows that for such channels $\mathcal{E}_{R}\left(\mathcal{D}_{(\eta, \alpha)}\right) \geq \mathcal{E}\left(\mathcal{D}_{(\eta, \alpha)}\right)=0$ (see Fig. 6).

Conclusion.-We reviewed the relation between quantum communication and entanglement distribution capacities, paying special attention to entanglement distribution assisted by classical feedback. By restricting ourselves to realistic protocols with a single final round of postprocessing, we defined the reverse entanglement distribution protocols. A subset of such protocols give an operational interpretation of the reverse coherent information, a symmetric counterpart of the coherent information. This allow us to define a new entanglement distribution capacity which is additive and outperforms the unassisted capacity for some important channels, such as the damping channel and its generalization.

We acknowledge financial support from the W. M. Keck Foundation Center for Extreme Quantum Information Theory. S. P. acknowledges financial support from the EU (Marie Curie fellowship).

[1] C. E. Shannon, Bell Syst. Tech. J. 27, 379 (1948).

[2] M. A. Nielsen and I.L. Chuang, Quantum Computation and Quantum Information (Cambridge University Press, Cambridge, England, 2002).

[3] B. Schumacher and M. A. Nielsen, Phys. Rev. A 54, 2629 (1996).

[4] D. P. DiVincenzo, P. W. Shor, and J. A. Smolin, Phys. Rev. A 57, 830 (1998); G. Smith and J. A. Smolin, Phys. Rev. Lett. 98, 030501 (2007); G. Smith and J. Yard, Science 321, 1812 (2008).

[5] S. Lloyd, Phys. Rev. A 55, 1613 (1997); I. Devetak, IEEE Trans. Inf. Theory 51, 44 (2005).

[6] I. Devetak and P. W. Shor, Commun. Math. Phys. 256, 287 (2005).

[7] T. M. Cover and J. A. Thomas, Elements of Information Theory (Wiley, New Jersey, 2006).

[8] A. W. Leung, Phys. Rev. A 77, 012322 (2008).

[9] F. Grosshans, G. van Assche, J. Wenger, R. Tualle-Brouri, and P. Grangier, Nature (London) 421, 238 (2003).

[10] I. Devetak and A. Winter, Phys. Rev. Lett. 93, 080501 (2004).

[11] V. Giovannetti and R. Fazio, Phys. Rev. A 71, 032314 (2005).

[12] H. Barnum, E. Knill, and M. A. Nielsen, IEEE Trans. Inf. Theory 46, 1317 (2000).

[13] C. H. Bennett, D. P. DiVincenzo, and J. A. Smolin, Phys. Rev. Lett. 78, 3217 (1997).

[14] D. Leung, J. Lim, and P. W. Shor, Phys. Rev. Lett. (to be published). 